\title{
Pernicious anemia presenting as glossitis
}

\author{
Akira Kobayashi MD, Hiromichi Iwasaki MD PhD
}

— Cite as: CMAJ 2020 April 20;192:E434. doi: 10.1503/cmaj.191331

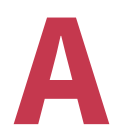

69-year-old Japanese woman presented with a 4-week history of painful tongue and reduced sense of taste. On physical examination, she had a smooth, red tongue without dorsal papillae, suggestive of glossitis (Figure $1 \mathrm{~A}$ ). Results from laboratory tests were consistent with macrocytosis without anemia: mean corpuscular volume 104.9 (normal range $80-97$ ) fL, hemoglobin 121 (normal range $110-165$ ) $\mathrm{mmol} / \mathrm{L}$, iron 10.92 (normal range 8.95-26.85) $\mu \mathrm{mol} / \mathrm{L}$, ferritin 72 (normal range 5-157) $\mu \mathrm{g} / \mathrm{L}$ and serum vitamin $B_{12} 77.49$ (normal range 147.6-442.8) pmol/L. In addition, an endoscopic biopsy of her gastric mucosa showed atrophic gastritis, and the result from a test for the presence of serum anti-intrinsic antibodies was positive. We diagnosed pernicious anemia.

Pernicious anemia is a macrocytic anemia caused by vitamin $B_{12}$ deficiency that results from a lack of intrinsic factor. Lack of intrinsic factor may be caused by atrophic gastritis and damage to the oxyntic mucosa and parietal cells, which normally produce hydrochloric acid and intrinsic factor. ${ }^{1}$ Glossitis presents in up to $25 \%$ of people with pernicious anemia, initially as bright red plaques that may evolve into atrophy of the lingual papillae. ${ }^{2}$ Oral manifestations of pernicious anemia, including glossitis and stomatitis, may occur in the absence of anemia and represent an early clinical sign of vitamin $\mathrm{B}_{12}$ deficiency. ${ }^{3}$ Other causes of glossitis include nutritional deficiencies of vitamin $B_{12}$, folic acid, riboflavin and niacin. ${ }^{4}$

We prescribed intramuscular methylcobalamin for our patient's pernicious anemia, and her painful tongue and reduced sense of taste resolved within days. Her tongue regained its normal appearance within a month (Figure 1B). She remains on maintenance therapy with intramuscular methylcobalamin.

\section{References}

1. Lahner E, Annibale B. Pernicious anemia: new insights from a gastroenterological point of view. World J Gastroenterol 2009;15:5121-8.

2. Graells J, Ojeda RM, Muniesa C, et al. Glossitis with linear lesions: an early sign of vitamin $\mathrm{B}_{12}$ deficiency. J Am Acad Dermatol 2009;60:498-500.

3. Field EA, Speechley JA, Rugman FR, et al. Oral signs and symptoms in patients with undiag nosed vitamin $B_{12}$ deficiency. J Oral Pathol Med 1995;24:468-70.

4. Reamy BV, Derby R, Bunt CW. Common tongue conditions in primary care. Am Fam Physician 2010;81:627-34.

\section{Competing interests:}

This article has been peer reviewed.

The authors have obtained patient consent.

Affiliations: Division of Internal Medicine (Kobayashi), Nanmei-kai Miyagami Hospital, Kagoshima, Japan; Department of Infection Control and Prevention (Iwasaki), University of Fukui, Fukui, Japan

Correspondence to: Akira Kobayashi, akirakobachan@msn.com

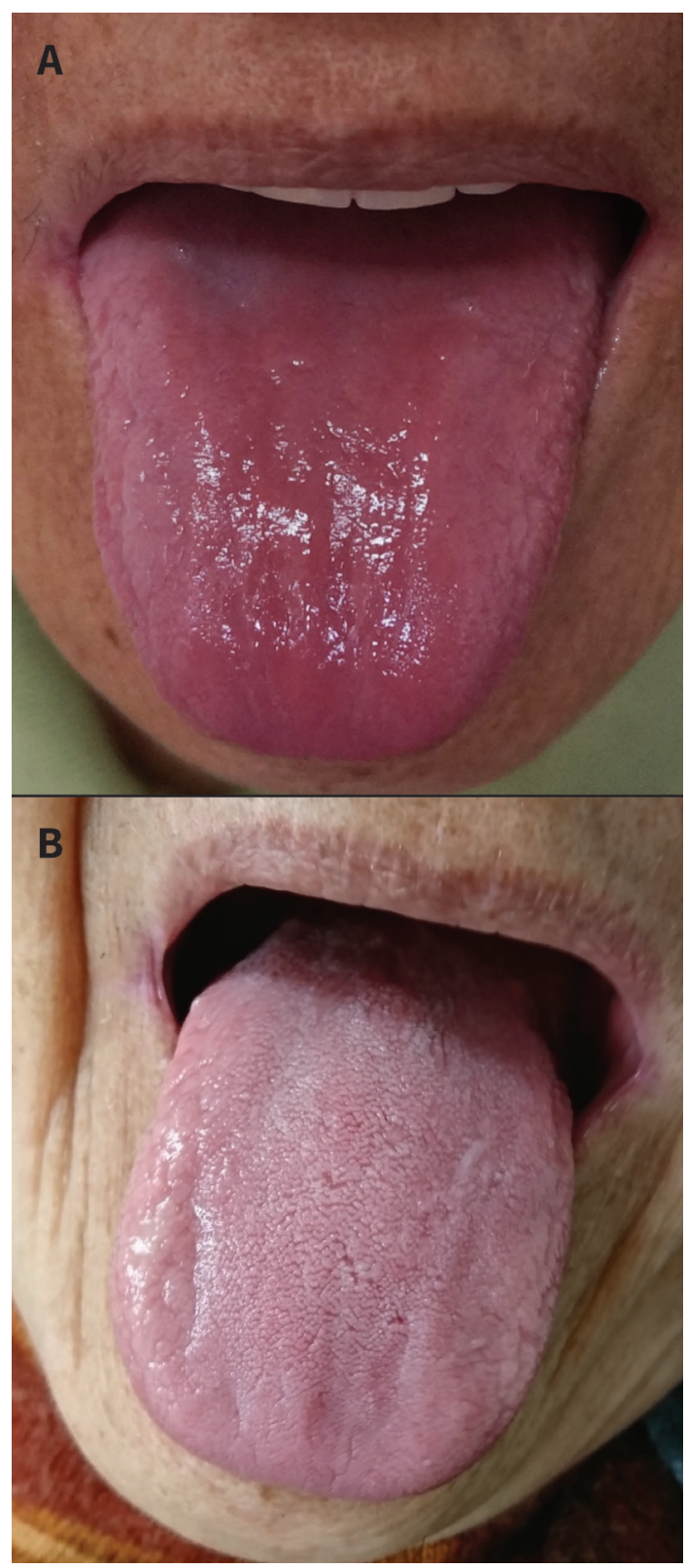

Figure 1: (A) View of the tongue of a 69-year-old woman in Japan with pernicious anemia presenting as glossitis (smooth and red dorsum of the tongue, without lingual papillae). (B) One month after starting treatment with methylcobalamin, her tongue appears normal. 